STOMACH

\title{
Novel action of gastric proton pump inhibitor on suppression of Helicobacter pylori induced angiogenesis
}

\author{
M Yeo, D-K Kim, S U Han, J E Lee, Y B Kim, Y K Cho, J H Kim, S W Cho, K-B Hahm

Background: Although activation of mitogen activated protein kinases (MAPKs) by Helicobacter pylori infection is associated with induction of host angiogenesis, which may contribute to $\mathrm{H}$ pylori associated gastric carcinogenesis, the strategy for its prevention has not been identified. As we previously reported a strong inhibitory action of gastric proton pump inhibitors (PPIs) on MAPK extracellular signal regulated kinase (ERK) $1 / 2$ phosphorylation, we investigated whether PPIs could suppress the $H$ pylori induced angiogenesis via inhibition of MAPK ERK $1 / 2$.

Methods: To address the relationship between $\mathrm{H}$ pylori infection and angiogenesis, comparative analysis of density of $\mathrm{CD} 34^{+}$blood vessel was performed in tissues obtained from $20 \mathrm{H}$ pylori positive gastritis and

See end of article for authors' affiliations

Correspondence to:

Professor M Yeo, Genome

Research Centre for

Gastroenterology, Ajou

University Medical Centre,

San 5, Wonchon-dong,

Yeongtong-gu, Suwon,

442-749, Korea;

marie8346@hotmail.com

Revised version received 14 June 2005

Accepted for publication 20 July 2005

Published online first

26 August 2005
$18 \mathrm{H}$ pylori negative gastritis patients. Expression of hypoxia inducible factor $1(\mathrm{HIF}-1 \alpha)$ and vascular endothelial growth factor (VEGF) was tested by reverse transcription-polymerase chain reaction and secretion of interleukin 8, and VEGF was measured by ELISA. To evaluate the direct effect of $H$ pylori infection on the tubular formation of human umbilical vein endothelial cells (HUVEC), an in vitro angiogenesis assay was employed. Activation of MAPK and nuclear factor $\kappa B(N F \kappa B)$ was detected by immunoblotting.

Results: $H$ pylori positive gastritis patients showed a higher density of CD34 $4^{+}$blood vessels (mean 40.9 (SEM 4.4)) than $H$ pylori negative gastritis patients (7.2 \pm 0.8 ), which was well correlated with expression of HIF-1 $\alpha$. Conditioned media from $H$ pylori infected gastric epithelial cells directly induced tubular formation of HUVEC and the increase of in vitro angiogenesis was suppressed by PPI treatment. Infection of $H$ pylori significantly upregulated expression of HIF- $1 \alpha$ and VEGF in gastric epithelial cells and expression of proangiogenic factors was mediated by MAPK activation and partially responsible for $N F \kappa B$ activation. PPIs effectively inhibited the phosphorylation of MAPK ERK1/2 that is a principal signal for $H$ pylori induced angiogenesis.

Conclusions: The fact that PPls could downregulate $H$ pylori induced angiogenesis indicates that antiangiogenic treatment using a PPI could be a promising protective therapeutic approach for $\mathrm{H}$ pylori associated carcinogenesis.
$\mathrm{T}$ hat chronic persistent gastric inflammation associated with Helicobacter pylori may play a crucial role in either the development or progression of gastric cancers has been generally agreed $^{1-3}$ but the exact molecular mechanisms of how longstanding $H$ pylori infection can induce cancer and make the procancer microenvironment favourable for the survival of tumour cells have not yet been clearly identified. The mechanisms fostering the neoplastic process of $H$ pylori infection have been revealed to include: (1) induction of neoplastic mutation by a considerable burden of oxidative stress $^{45}$; (2) imbalance between cell proliferation and apoptosis $^{67}$; (3) production of proteases and growth factors providing the environment for cell migration ${ }^{8-10}$; and (4) induction of host angiogenesis. ${ }^{11-15}$

Among the diverse host cellular responses related to $H$ pylori associated inflammation or carcinogenesis, some investigators reported that angiogenic growth factors induced by $H$ pylori might be primarily important. ${ }^{11-15}$ Kitadai and colleagues $^{11}$ and Cox and colleagues ${ }^{12}$ found that $H$ pylori infection induced several angiogenic factors and proteases, such as interleukin 8 (IL-8), vascular endothelial growth factor (VEGF), angiogenin, urokinase-type plasminogen activator, and metalloprotease 9 using high throughput technology of cDNA microarray analysis. Strowski and colleagues $^{13}$ also reported that $H$ pylori stimulated host VEGF gene expression via a mitogen activated protein kinase
(MAPK) pathway. These data imply that $H$ pylori are capable of inducing host angiogenesis, which may play a critical role in the development and progression of gastric cancer. However, trials documenting the precise mechanism and preventive therapeutic approaches have not been performed.

The $\mathrm{H}^{+} / \mathrm{K}^{+}$-ATPase of gastric parietal cells exchanges luminal $\mathrm{K}^{+}$for cytoplasmic $\mathrm{H}^{+}$and is the enzyme primarily responsible for gastric acidification. ${ }^{16}{ }^{17}$ The enzyme consists of two subunits, a $114 \mathrm{kDa} \alpha$ subunit and a $35 \mathrm{kDa} \beta$ subunit. The $\alpha$ subunit containing ATP and cation binding sites carries out the catalytic and transporting function of the proton pump. The heavily glycosylated $\beta$ subunit is required for endocytic retrieval of the $\mathrm{H}^{+} / \mathrm{K}^{+}$-ATPase from the canalicular membranes and is also essential for protecting the proton pump from the acid milieu environment. As abnormally controlled gastric acid causes several gastrointestinal acid related diseases, including gastro-oesophageal reflux disease, gastric ulcer, duodenal ulcer, and Barrett's oesophagus,

Abbreviations: PPI, proton pump inhibitor; PPZ, pantoprazole; HIF- $1 \alpha$, hypoxia inducible factor 1; VEGF, vascular endothelial growth factor; IL-8, interleukin 8; MAPKs, mitogen activated protein kinases; ERK, extracellular signal regulated kinase; $N F \kappa B$, nuclear factor $\kappa B$; RT-PCR, reverse transcription-polymerase chain reaction; HUVEC, human umbilical vein endothelial cells; PBS, phosphate buffered saline; CFU, colony forming units; PDTC, 1-pyrrolidinecarbodithioic acid; ELISA, enzyme linked immunosorbent assay 
gastric proton pump inhibitors (PPIs) have been developed for the treatment of these acid related diseases. ${ }^{18-20}$ Among these, pantoprazole, a substituted 2-pyridyl methyl/sulfinyl benzimidazole derivative, is a prodrug requiring protonation for functional activation under acidic conditions, accumulates selectively in acidic gastric luminal space, and ultimately inhibits acid secretion by covalent binding with cysteine residues in the $\alpha$ subunit of $\mathrm{H}^{+} / \mathrm{K}^{+}$-ATPase. ${ }^{18-20}$ The last decade has seen standardisation of the treatment regimens for $H$ pylori eradication, with the use of triple therapy consisting of a PP) and two antibiotics, mainly clarithromycin and amoxicillin. Blockage of gastric acid secretion by PPIs contributes towards eradication of $H$ pylori via the rising $\mathrm{pH}$ of the gastric lumen. Appropriately high $\mathrm{pH}$ values increase antimicrobial susceptibility of $H$ pylori because the minimum inhibitory concentration of most antibiotics against $H$ pylori is very dependent on the $\mathrm{pH}$ of the environment. ${ }^{21-26}$

Previously, we found that PPIs could exercise selective induction of apoptosis in gastric cancer cells, which was due to a significant inhibitory action of PPIs on MAPK activation. ${ }^{27}$ As Strowski and colleagues ${ }^{13}$ reported that $H$ pylori stimulated host VEGF gene expression via the MAPK pathway, we hypothesised that PPIs could exert antiangiogenesis actions through MAPK inhibition in $H$ pylori induced angiogenesis. Here, we have found that infection with $H$ pylori significantly upregulated angiogenesis of the gastric mucosa by strong induction of proangiogenic factors, including IL-8, hypoxia inducible factor 1 (HIF-l $\alpha$ ), and VEGF and, remarkably, angiogenesis induced by $H$ pylori was attenuated by PPI treatment.

\section{MATERIALS AND METHODS Tissue samples}

Biopsied samples were obtained from five patients (mean age 48 years) with functional dyspepsia without $H$ pylori infection, 20 patients (mean age 55 years) with chronic active $H$ pylori positive gastritis, and 18 patients (mean age 54 years) with $H$ pylori negative gastritis induced mostly by nonsteroidal anti-inflammatory drugs or other causes during gastroscopy. The presence of $H$ pylori was determined using the following tests: haematoxylin-eosin staining and Giemsa staining of biopsied tissues, rapid urease test, and urea breath test. When all of the above were negative, the case was defined as $H$ pylori negative and if more than two of these tests were positive, the case were defined as $H$ pylori positive. Gastritis was evaluated histologically and scored according to a modified Sydney classification ${ }^{28}$; two different pathologists scored the degree of gastritis independently. Informed written consent was obtained from patients and the study was approved by Institutional Review Board.

\section{Immunohistochemistry for counting vessels in the gastric mucosa}

Immunohistochemical staining of $\mathrm{CD}_{3} 4^{+}$endothelial cells was performed to analyse the degree of angiogenesis in the gastric mucosa of gastritis patients. For immunohistochemical detection, $10 \%$ buffered formalin fixed paraffin embedded sections were deparaffinised, rehydrated, and then boiled in $100 \mathrm{mM}$ Tris buffered saline ( $\mathrm{pH}$ 7.6) with $5 \%$ urea in an $850 \mathrm{~W}$ microwave oven for five minutes, followed by two more treatments of five minutes each. Then sections were stained with Histostain-Plus kit (Zymed Laboratories Inc., San Francisco, California, USA) according to the manufacturer's instructions. Primary antibodies against the CD34 endothelial cell marker were purchased from Novocastra Laboratories (clone QBEnd/10; UK). Sections were counterstained with haematoxylin. CD34 positive blood vessels were counted on three separate sites
( $\times 100$ magnified field) and presented as mean (SEM) of $20 \mathrm{H}$ pylori positive and $18 \mathrm{H}$ pylori negative cases.

\section{Cell culture, bacteria strain, and reagents}

Human gastric epithelial AGS cells were cultured in RPMI 1640 medium (Gibco BRL, Grand Island, New York. USA) containing $10 \%$ fetal bovine serum and $100 \mathrm{U} / \mathrm{ml}$ penicillin in a humidified 5\% $\mathrm{CO}_{2}$ atmosphere. Human umbilical vein endothelial cells (HUVEC) were obtained by collagenase treatment of umbilical cord veins, as previously described. ${ }^{29}$ Cells were cultured on gelatin coated dishes and propagated in RPMI 1640 medium supplemented with $20 \%$ bovine calf serum, $90 \mu \mathrm{g} / \mathrm{ml}$ heparin (Sigma Chemical Co, St Louis, Missouri, USA), and $50 \mu \mathrm{g} / \mathrm{ml}$ endothelial cell growth factor. A $c a g A^{+}$and $v a c A^{+}$standard strain of $H$ pylori (ATCC 43504) was obtained from the American Type Culture Collection (ATCC, Manassas, Virginia, USA). H pylori were recovered from frozen stock by seeding on a blood agar plate including $7 \%$ sheep blood at $37^{\circ} \mathrm{C}$ for five days under microaerophilic conditions $\left(5 \% \mathrm{O}_{2}, 10 \% \mathrm{CO}_{2}\right)$ generated with campy pouch (Becton Dickinson Microbiology Systems, Sparks, Maryland, USA). For inoculation of the bacteria, $H$ pylori were resuspended in phosphate buffered saline (PBS) to an $\mathrm{A}_{450}$ of 1.2 units, which corresponds to a bacterial concentration of $5 \times 10^{8}$ colony forming units $(\mathrm{CFU}) / \mathrm{ml}$, and cocultured with AGS cells at a concentration of $5 \times 10^{7} \mathrm{CFU} / \mathrm{ml}$.

A solution of pantoprazole (PPZ) was obtained from Altana Pharma AG (Konstanz, Germany). PD098059 (50 $\mu$ M, extracellular signal regulated kinase (ERK) $1 / 2$ inhibitor; Cell Signaling Technology, Beverly, Massachusetts, USA), SB203580 $(10 \mu \mathrm{M}, \quad$ p38 inhibitor; Cell Signaling Technology), 1-pyrrolidinecarbodithioic acid (PDTC $100 \mu \mathrm{M}$,

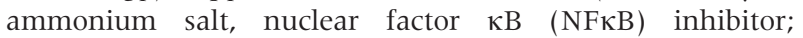
Calbiochem, La Jolla, California, USA), and BAY11-7082 ( $5 \mu \mathrm{M}, \mathrm{NF \kappa B}$ inhibitor; Calbiochem) were used in the cell culture experiments. Briefly, to evaluate the effect of these inhibitors or PPIs, they were preincubated with AGS cells for eight hours, washed with PBS, and inoculated with $H$ pylori.

\section{Western blot analysis}

Human gastric epithelial AGS cells $\left(1 \times 10^{7}\right.$ cells $/ 100 \mathrm{~mm}^{2}$ culture dish) were incubated with $0,100,200$, or $400 \mu \mathrm{M}$ PPI for eight hours, washed with PBS three times, and then inoculated with $H$ pylori $\left(5 \times 10^{7} \mathrm{CFU} / \mathrm{ml}\right)$ for 15 minutes (western blotting for ERKl/2) or two hours (western blotting for $N F \kappa B)$. Cells were resuspended in lysis buffer $(20 \mathrm{mM}$ Tris, pH 7.5, $150 \mathrm{mM} \mathrm{NaCl}, 1 \%$ Triton X-100, 1 mM EDTA, I $\mathrm{mM}$ EGTA, and protease inhibitor cocktail; Roche, Mannheim, Germany). The suspension was sonicated for approximately 30 seconds and centrifuged at $15000 \mathrm{~g}$ for 30 minutes. For documenting NFKB activation, nuclear/ cytosolic fractionation was performed using NE-PER Nuclear and Cytoplasmic Extraction Kit (Pierce, Rockford, Illinois, USA) following the manufacturer's protocol. Isolated protein $(20 \mu \mathrm{g})$ was subjected to western blotting. Proteins were extracted from the cells, electrophoresed on $12 \%$ sodium dodecyl sulphate-polyacrylamide gels, and transferred to PVDF membranes using a semidry transfer system (Hoeffer Phamacia Biotech, San Francisco, California, USA). Membranes were blocked in 5\% non-fat dry milk and probed with 1:1000 dilution of specific antibodies corresponding to phospho-ERK (E-4), total ERK (K-23), NFкB p65 (F-6), or $\alpha$-tubulin (TU-02); all antibodies were purchased from Santa Cruz Biotechnology (Santa Cruz, California, USA).

\section{Reverse transcription-polymerase chain reaction (RT-PCR) analysis}

Total RNA was isolated from cells with appropriate treatment using TRIzol reagent (Life Technologies, Milan, Italy), and 
A

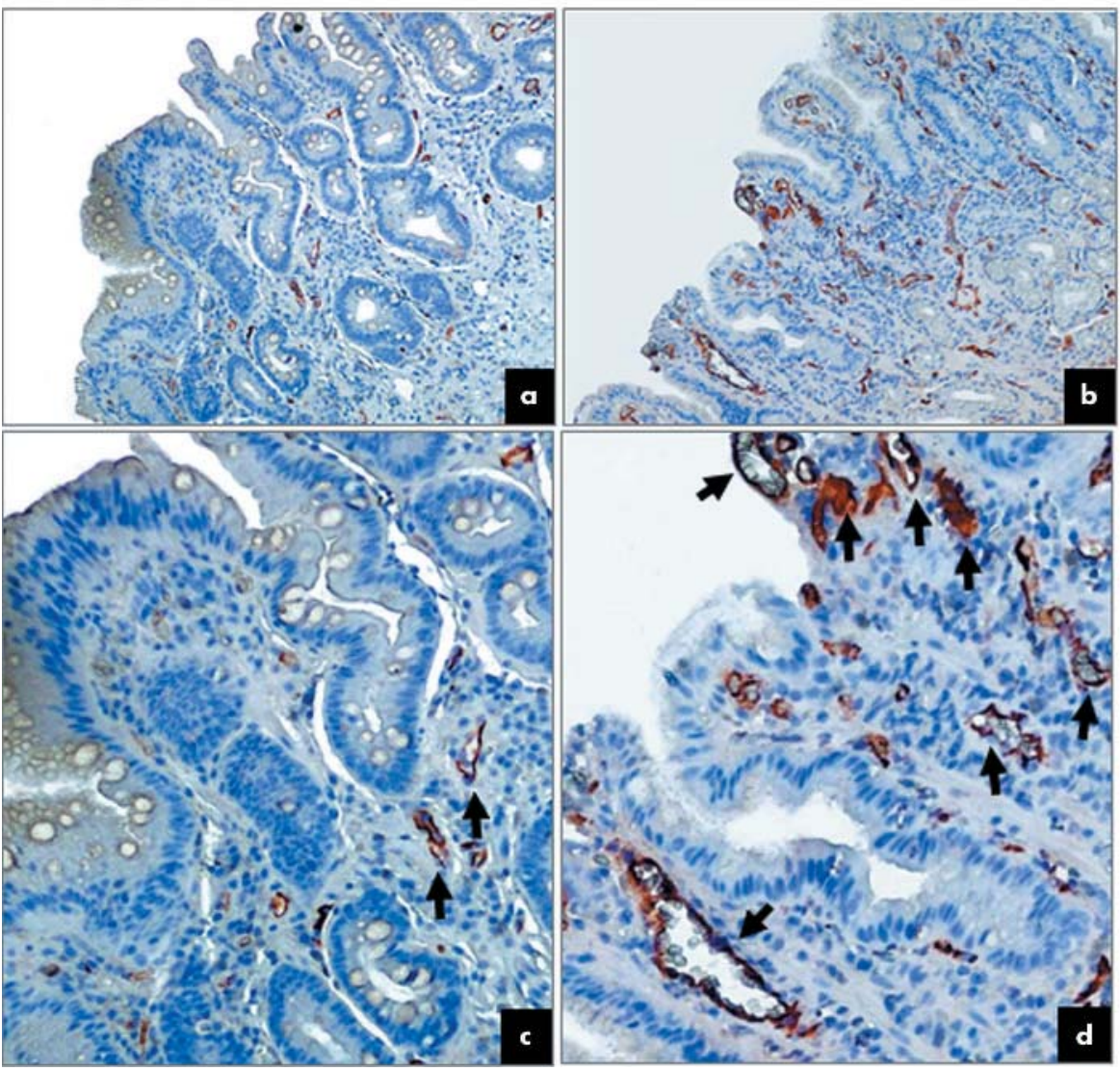

B

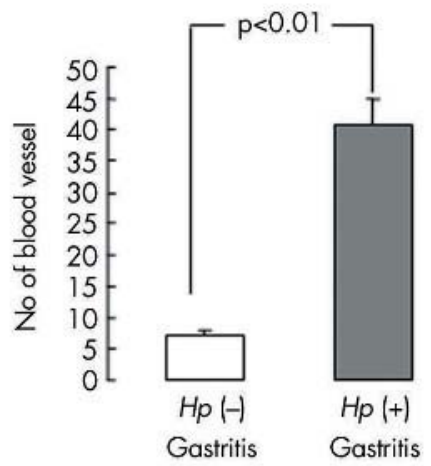

Figure 1 Immunohistochemical staining of the CD34 endothelial cell marker. (A) Immunohistological analysis of CD34 positive blood vessels in gastric biopsy specimens of Helicobacter pylori negative $(a, 100 x ; c, 200 x)$ and $H$ pylori positive $(b, 100 x ; d, 200 \times)$ gastritis. Specimens obtained from $H$ pylori negative gastritis or $\mathrm{H}$ pylori positive gastritis during endoscopic examination were used for immunohistological analysis with anti-CD34 antibodies. CD34 ${ }^{+}$blood vessels were stained by a dark red colour (arrow), counterstained with haematoxylin showing a blue colour. (B) Mean number of blood vessels stained with CD34. Number of blood vessels with equal dimensions were counted in triplicate for each sample and are represented as means (SD). There was a statistically significant difference between the two groups $(p<0.01)$. Hp, Helicobacter pylori.

$2 \mu \mathrm{g}$ total RNA were reverse transcribed according to the manufacturer's instructions (M-MLV reverse transcriptase; Promega, Madison, Wisconsin, USA). PCR was performed using the Premix Ex Taq kit (Takara, Chiba, Japan) with specific primers as follows: 5'-CTC AAA GTC GGA CAG CCT
CA $-3^{\prime}$ and $5^{\prime}$-CCC CGC AGT AGG TTT CTG CT- $3^{\prime}$ for $H I F-1 \alpha$; 5'-TCG GGC CTC CGA AAC CAT G-3' and 5'-GGT TCC CGA AAC CCT GAG G -3' for VEGF; and 5'-TTG TTG CCA TCA ATG ACC CC-3' and 5'-TGA CAA AGT GGT CGT TGA GG-3' for $G A P D H$. The PCR reaction was carried out for 28 thermal
A

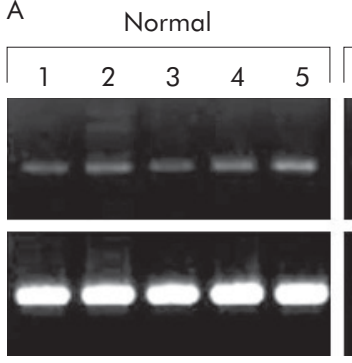

$\mathrm{Hp}^{-}$gastritis

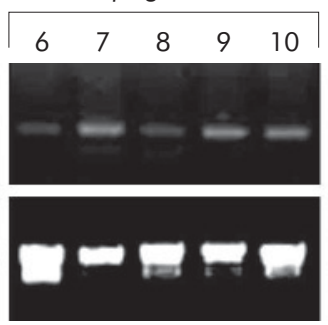

$\mathrm{Hp}^{+}$gastritis

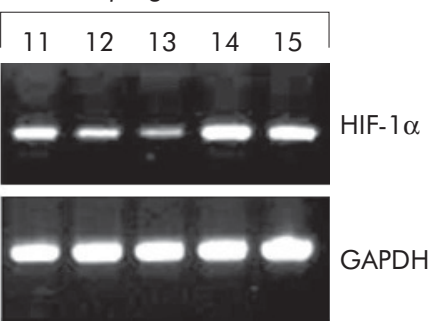

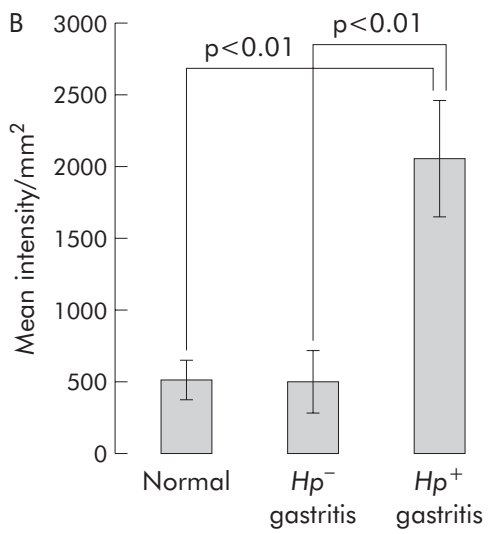

Figure 2 Expression of hypoxia inducible factor 1 (HIF-1 $1 \alpha$ mRNA in human gastric mucosa. (A) Reverse transcription-polymerase chain reaction of HIF- $1 \alpha$ was done with total RNA isolated from Helicobacter pylori $(H p)$ negative gastritis $(n=5), H$ pylori positive gastritis $(n=5)$, and normal stomachs $(\mathrm{n}=5)$. (B) Relative expression of HIF- $1 \alpha \mathrm{mRNA}$ is represented as mean intensity $/ \mathrm{mm}^{2}$. HIF- $1 \alpha$ expression was significantly higher in gastric mucosa of $\mathrm{H}$ pylori positive gastritis than in $\mathrm{H}$ pylori negative gastritis in spite of similar expression of GAPDH $(\mathrm{p}<0.01)$. 
cycles of $94^{\circ} \mathrm{C}$ for one minute at $55^{\circ} \mathrm{C}$ (for HIF- $1 \alpha$ and GAPDH) or $60^{\circ} \mathrm{C}$ (for VEGF) for one minute, and $72^{\circ} \mathrm{C}$ for one minute. The product was resolved on $1 \%$ agarose gel and stained with ethidium bromide.

\section{Enzyme linked immunosorbent assay (ELISA)}

Immunoreactive human IL-8 and VEGF were measured in culture supernatants of AGS cells using enzyme linked immunosorbent assay (ELISA) kits according to the manufacturer's instructions (HyCult Biotechnology, Uden, the Netherlands). AGS cells were grown in six well cell culture dishes, incubated in the presence or absence of PPI for eight hours, and after washing with PBS, cocultured with $H$ pylori for various times. Culture supernatant $(200 \mu \mathrm{l})$ was used for analysis of IL-8 and VEGF production.

\section{In vitro angiogenesis assay}

In vitro angiogenesis assay was slightly modified from Kitadai and colleagues. ${ }^{11}$ Briefly, AGS cells $\left(1 \times 10^{7}\right.$ cells/ $100 \mathrm{~mm}^{2}$ culture dish) were incubated with $0,100,200$, or $400 \mu \mathrm{M}$ PPI for eight hours, washed with PBS three times, and then inoculated with $H$ pylori $\left(5 \times 10^{7} \mathrm{CFU} / \mathrm{ml}\right)$ for 24 hours. The cell culture supernatant was harvested and centrifuged at $5000 \mathrm{~g}$ for 30 minutes. Conditioned media was prepared by $1: 1$ dilution of the culture supernatant with HUVEC endothelial cell medium. The conditioned media were filtered through $0.4 \mu \mathrm{M}$ pore filters (Millipore, Boston, Massachusetts, USA) to remove $H$ pylori and then the media were added to HUVEC culture and changed every three days. After nine days, the HUVEC were observed for tubular formation under microscopy and confirmed expression of the endothelial cell maker, CD31, by immunocytofluorescence staining. HUVEC were fixed in ice cold methanol for five minutes, frozen at $-20^{\circ} \mathrm{C}$, and treated with $1 \%$ Triton $\mathrm{X}-100$ / PBS for 10 minutes at room temperature. Cells were blocked with 5\% bovine serum albumin for 30 minutes and probed with anti-CD31 antibodies (1:100 diluted in 5\% bovine serum albumin; Santa Cruz Biotechnology) for two hours. Cy3 conjugated secondary antibodies were used to visualise under a inverted fluorescence microscope (Olympus, IX71, Tokyo, Japan).

\section{Statistics}

All values are expressed as mean (SEM) and the MannWhitney U test and Friedman ANOVA test were used for statistical calculations.

\section{RESULTS}

Distinct expression of CD34 ${ }^{+}$blood vessels between $\mathrm{H}$ pylori positive and negative gastritis patients

Stomach tissue samples were obtained from gastritis patients during endoscopy examination and were evaluated histologically by haematoxylin-eosin staining. Finally, we choose $20 \mathrm{H}$ pylori positive gastritis and $18 \mathrm{H}$ pylori negative gastritis cases with a similar degree of gastritis, scored according to the modified Sydney classification, ${ }^{28}$ as the degree of gastric inflammations itself can affect angiogenesis. Immunohistochemical staining using antibodies against the CD34 endothelial cell marker was performed to evaluate the difference in angiogenesis according to $H$ pylori infection. The results showed that patients with $H$ pylori positive gastritis (fig lA (b, d)) showed significantly higher expression of CD34 positive blood vessels in the gastric mucosa layer than that of patients with $H$ pylori negative gastritis (fig lA $(\mathrm{a}, \mathrm{c})$ ). The number of blood vessels was counted in three sites, for each specimen, with equal dimensions, and mean levels are shown in fig $1 \mathrm{~B}$. While $H$ pylori negative gastritis samples had a mean of 7.2 (SEM 0.8) blood vessels per $\times 100$ magnified field, $H$ pylori positive gastritis samples had a significant increased number (mean 40.9 (SEM 4.4)) of blood vessels $(\mathrm{p}<0.01)$. Moreover, blood vessels observed in cases with $H$ pylori positive gastritis (fig lA (b, d), arrow) were thicker and larger than those of $H$ pylori negative cases (fig lA (a, c), arrow). Interestingly, blood vessels were found more abundantly in the mesenchymal stromal layer below the mucosa layer but the number of blood vessels in the stromal layer was not different between $H$ pylori positive gastritis and $H$ pylori negative gastritis cases, suggesting that $H$ pylori infection may
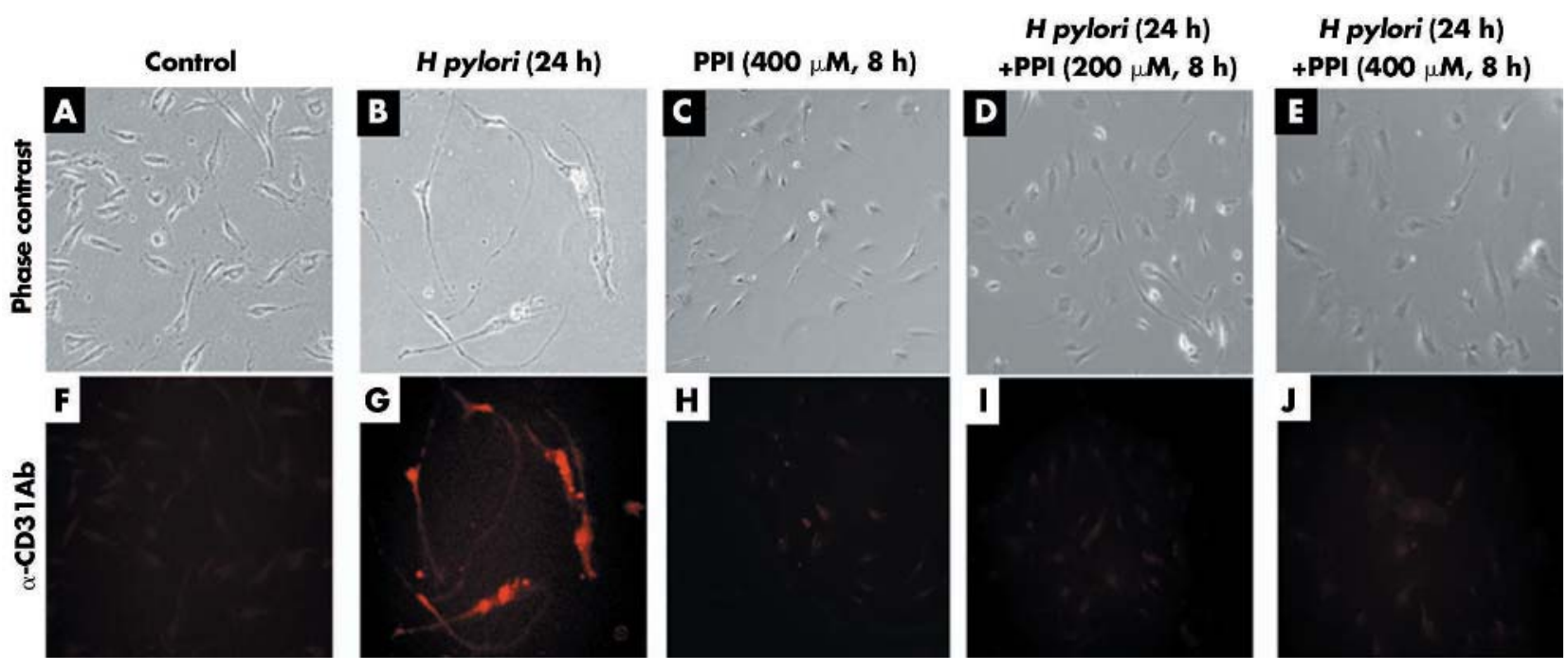

Figure 3 In vitro angiogenesis assay. AGS cells $\left(1 \times 10^{7}\right.$ cells $/ 100 \mathrm{~mm}^{2}$ culture dish) were incubated with 0,200 , or $400 \mu \mathrm{M}$ proton pump inhibitor (PPI) for eight hours, washed with phosphate buffered saline three times, and inoculated with Helicobacter pylori $\left(5 \times 10^{7} \mathrm{CFU} / \mathrm{ml}\right)$ for 24 hours. Conditioned media were prepared from 1:1 dilution of the cell culture supernatant and the human umbilical vein endothelial cell (HUVEC) medium. Conditioned media were filtered through a $0.4 \mu \mathrm{M}$ pore filter to remove $H$ pylori and then added to the HUVEC culture which was changed every three days. After nine days, HUVEC were observed in a tubular formation under microscopy (A-E) and expression of the endothelial cell maker, CD31, was confirmed by immunocytofluorescence staining (F-J). (A, F) Control HUVEC cells; (B, G) HUVEC cells incubated with conditioned media of $H$ pylori infected AGS; (C, H) HUVEC cells incubated with conditioned media of $400 \mu \mathrm{M}$ PPI treated AGS; (D, I) HUVEC cells incubated with conditioned media of $200 \mu \mathrm{M} \mathrm{PPI} / \mathrm{H}$ pylori infected AGS; (E, J) HUVEC cells incubated with conditioned media of $400 \mu \mathrm{M} \mathrm{PPI} / \mathrm{H}$ pylori infected AGS. 

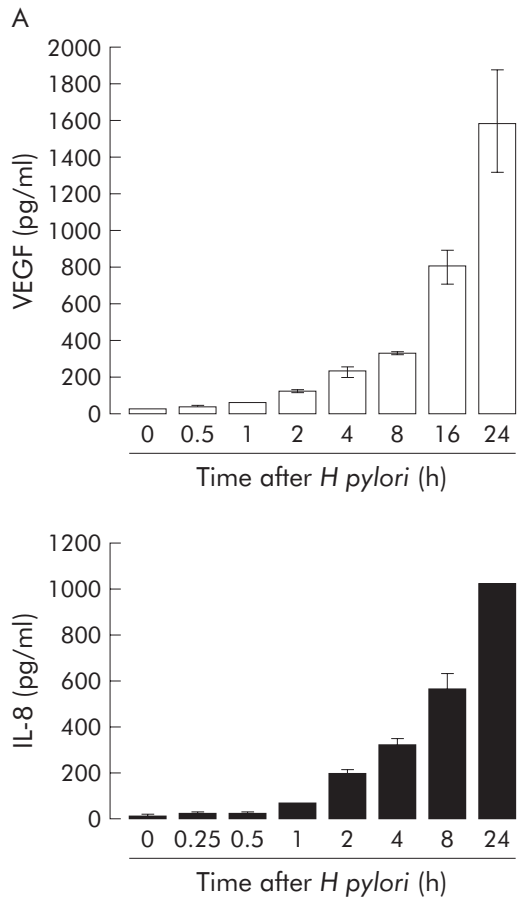
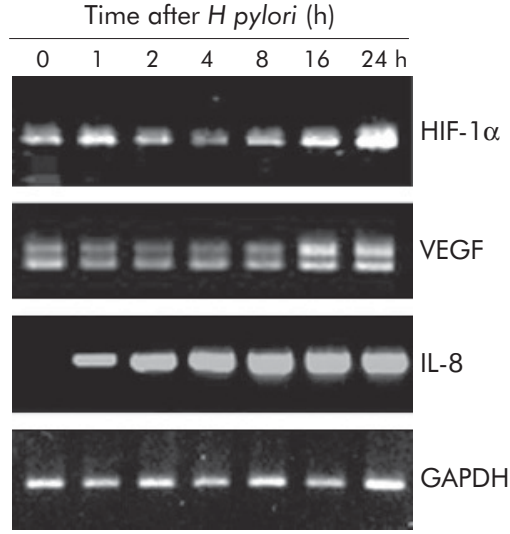

$\mathrm{C}$
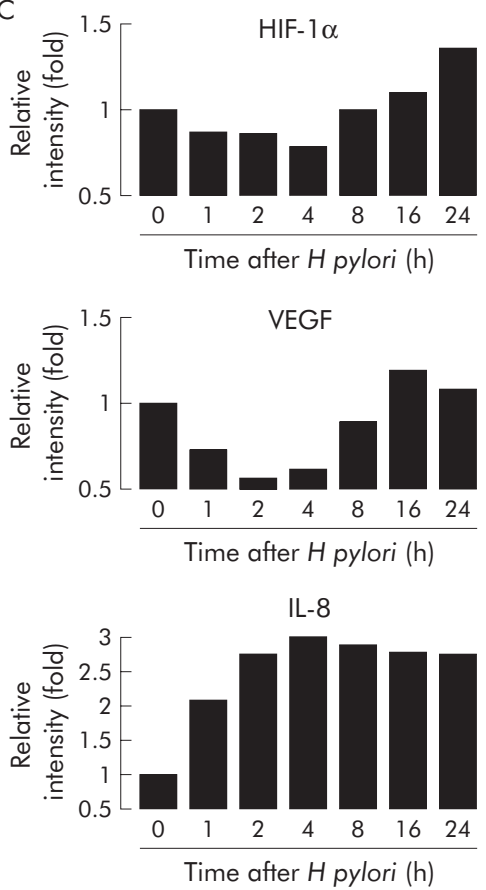

Figure 4 Release and expression of vascular endothelial growth factor (VEGF) and interleukin 8 (IL-8) from Helicobacter pylori infected AGS cells. (A) Production of VEGF (top) and IL-8 (bottom) was measured by ELISA with the culture supernatant of AGS cells infected with $H$ pylori for the indicated times. (B) Induction of hypoxia inducible factor 1 (HIF-1 $\alpha$ ), VEGF, and IL-8 mRNA by $H$ pylori infection was tested by reverse transcription-polymerase chain reaction analysis in AGS cells incubated with the bacterium for the indicated times. (C) Relative band intensity is presented as fold ratio.

be associated with induction of angiogenesis in $H$ pylori infected gastric mucosa.

We then evaluated if there were any differences in expression of HIF- $1 \alpha$, the potent angiogenic transcriptional factor (fig 2). As a control, we used gastric biopsies obtained from five cases diagnosed with functional dyspepsia with no significant abnormal gastroscopic findings and no $H$ pylori infection. Compared with HIF- $1 \alpha$ mRNA from five normal stomachs, expression was not altered in five $H$ pylori negative gastritis but was significantly increased in $H$ pylori positive gastritis $(\mathrm{p}<0.01)$, suggesting that HIF- $1 \alpha$ is responsible for $H$ pylori induced angiogenesis (fig $2 \mathrm{~B}$ ).

\section{Suppression of $H$ pylori induced in vitro angiogenesis by gastric PPI}

To prove a direct effect of $H$ pylori infection on angiogenesis, we performed an in vitro angiogenesis assay. Conditioned media obtained from $H$ pylori infected AGS cells were added to HUVEC culture flasks and morphological changes in the endothelial cells were observed. After nine days, HUVEC became long in shape and formed a tubular structure (fig 3B) compared with conditioned media of non- $H$ pylori infected AGS (fig 3A). CD31 immunofluorescence staining showed a dense intensity of CD31 molecules in HUVEC cells incubated with the culture supernatants of $H$ pylori infected AGS (fig 3G) while control media obtained from non- $H$ pylori infected AGS stimulated neither tubular formation of HUVEC nor expression of the CD31 endothelial cell marker (fig 3A, F).

Results of in vitro angiogenesis assay strongly suggested that $H$ pylori infection stimulated infected gastric epithelial cells to secrete proangiogenic factors which induce growth and differentiation of endothelial HUVEC. Interestingly, pretreatment with PPI ( $200 \mu \mathrm{M}$ or $400 \mu \mathrm{M}$, for eight hours) on AGS cells prior to $H$ pylori inoculation significantly inhibited tubular formation (fig 3D, E) and CD31 expression of endothelial HUVEC (fig 3I, J). However, no significant changes were noted in HUVEC incubated with $400 \mu \mathrm{M}$ PPI alone (fig 3C, H), suggesting that PPI itself did not influence tubular formation of HUVEC. The data clearly indicate that PPI suppressed $H$ pylori induced in vitro angiogenesis, suggesting that antiangiogenic treatment with PPI could be a promising therapeutic approach for $H$ pylori associated carcinogenesis.

\section{Production of proangiogenic factors from $\mathrm{H}$ pylori} infected gastric epithelium and its inhibition by PPI

Following $H$ pylori infection, AGS cells significantly secreted VEGF and IL-8, well characterised as proangiogenic factors, in a time dependent manner (fig 4A). Maximal induction of IL-8 (mean 1019 (SEM 278) pg/ml) and VEGF (1597 (94) $\mathrm{pg} / \mathrm{ml}$ ) was observed after 24 hours of inoculation (fig 4A). We also examined mRNA expression of these angiogenic factors using RT-PCR analysis (fig 4B). Expression of VEGF mRNA, one of the HIF- $1 \alpha$ target genes, was induced after 16 hours of $H$ pylori infection, showing the correlation with HIF- $1 \alpha$ expression (fig $4 \mathrm{~B}$ ). IL-8 mRNA was also significantly induced after $H$ pylori infection (fig 4B). All of these results suggest that synthesis of angiogenic growth factors is stimulated by $H$ pylori infection in gastric epithelial cells, which could induce proliferation and differentiation of endothelial cells.

Because PPI showed a strong antiangiogenic action in the in vitro angiogenesis assay (fig 3), we measured the effect of PPI on expression of these angiogenic factors (fig 5). Secretion of IL-8 in the supernatants of $H$ pylori infected AGS cells was found to be remarkably suppressed after PPI treatment in a dose dependent manner (fig 5A). Following eight hours of infection with $H$ pylori, IL-8 production increased up to $870 \mathrm{pg} / \mathrm{ml}$ but this increment in IL-8 production was significantly attenuated by PPI pretreatment. Pretreatment with PPI showed a considerable regulatory effect on $H$ pylori mediated VEGF synthesis (fig 5A). Suppression of these angiogenic factors by PPI was evidenced 

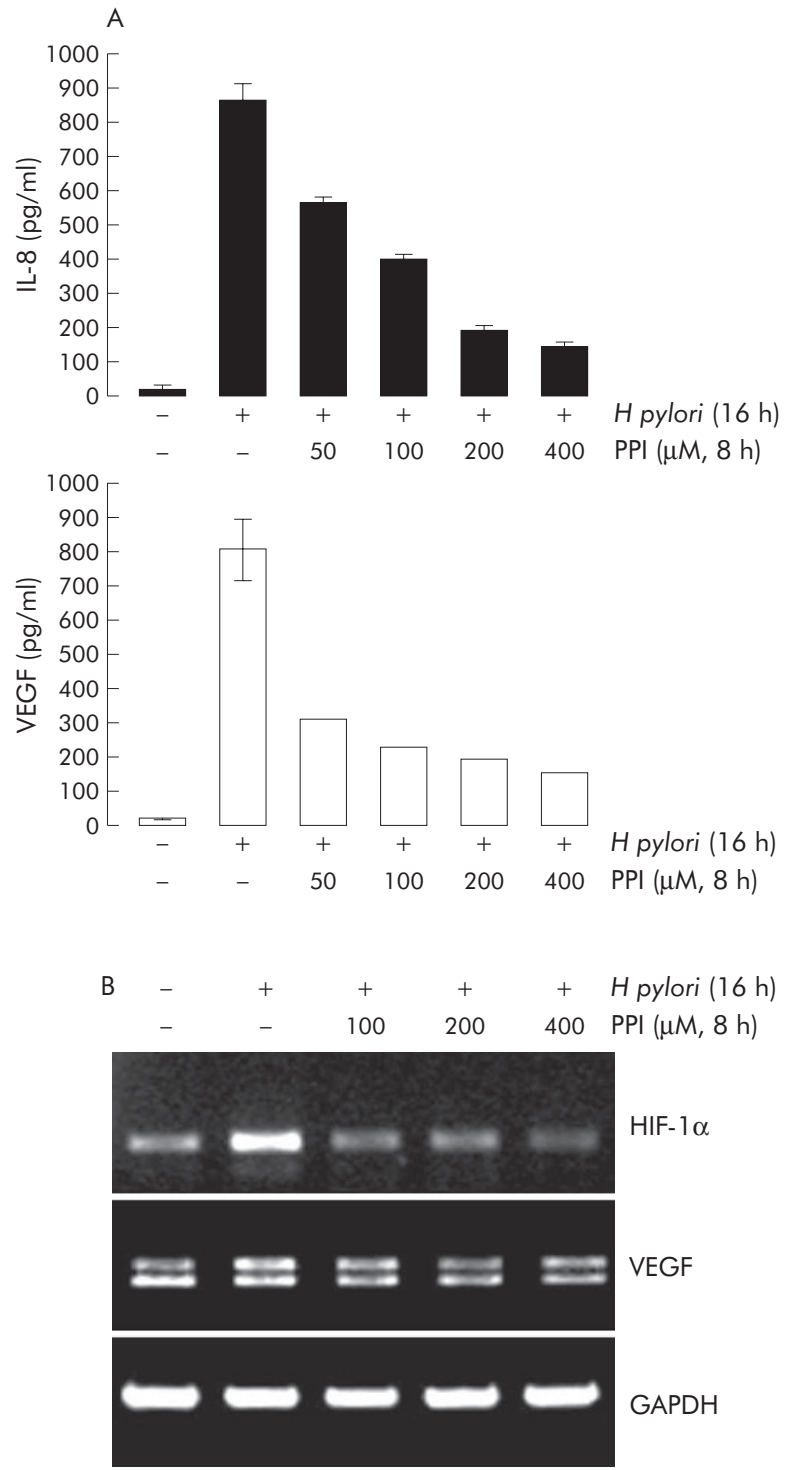

Figure 5 (A) Effects of proton pump inhibitor (PPI) on expression of angiogenic growth factors interleukin 8 (IL-8), hypoxia inducible factor 1 (HIF-1 $\alpha$ ), and vascular endothelial growth factor (VEGF). To examine the inhibitory effect of PPI on Helicobacter pylori induced angiogenic growth factor expression, AGS cells $\left(1 \times 10^{7}\right.$ cells $/ 100 \mathrm{~mm}^{2}$ culture dish) were incubated with $0,50,100,200$, or $400 \mu \mathrm{M}$ PPI for eight hours, washed with phosphate buffered saline three times, and inoculated with $H$ pylori $\left(5 \times 10^{7} \mathrm{CFU} / \mathrm{ml}\right)$ for 16 hours. Production of IL-8 (top) and VEGF (bottom) was measured in culture supernatants of the cells by ELISA. (B) Total RNA extracted from cells was used in the reverse transcriptionpolymerase chain reaction analysis of HIF- $1 \alpha$ and VEGF.

by transcriptional inhibition of the genes (fig 5B). At $400 \mu \mathrm{M}$ of PPI, expression of VEGF and HIF-l $\alpha$ seemed to decline relevant to that of control AGS cells.

\section{Expression of $H$ pylori induced angiogenic factors is} mediated by activation of ERK $1 / 2$

As VEGF and IL-8 expression was found to be regulated by MAPK and NFKB signalling, we assessed the involvement of MAPK ERKl/2 and NFKB on $H$ pylori induced angiogenesis using specific inhibitors (fig 6). PD098059 (50 $\mu \mathrm{M}$ ), one of the ERK inhibitors, strongly inhibited $H$ pylori induced HIF$1 \alpha$ and VEGF expression, and SB203580 $(10 \mu \mathrm{M})$, a p38 inhibitor, was also able to inhibit expression of these angiogenic factors. PDTC (ammonium salt, $100 \mu \mathrm{M}$ ) a

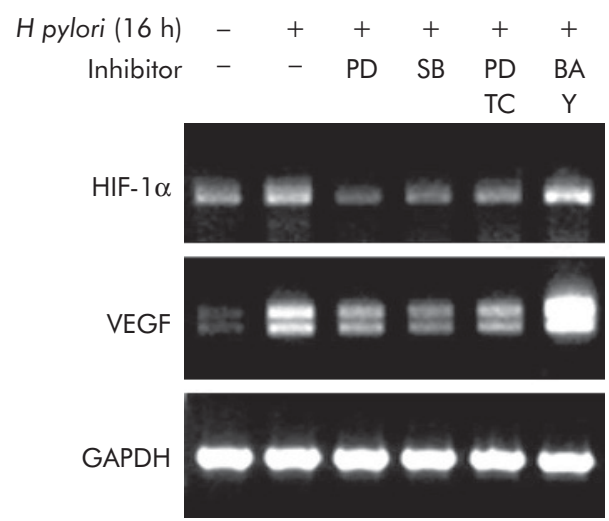

Figure 6 Involvement of mitogen activated protein kinase and nuclear factor $\kappa B(N F \kappa B)$ in Helicobacter pylori induced mRNA expression of hypoxia inducible factor 1 (HIF-1 $\alpha$ ) and vascular endothelial growth factor (VEGF). Prior to inoculation with $\mathrm{H}$ pylori, AGS cells were treated with each inhibitor (50 $\mu \mathrm{M}$ PD098059, $10 \mu \mathrm{M}$ SB203580, $100 \mu \mathrm{M}$ PDTC, or $5 \mu \mathrm{M}$ BAY1 1-7082 (BAY)) for eight hours, and their effects on $H$ pylori induced HIF- $1 \alpha$ and VEGF expression were evaluated by reverse transcription-polymerase chain reaction. PD, PD098059, extracellular signal regulated kinase (ERK) $1 / 2$ inhibitor; SB, SB203580, p38 inhibitor; PDTC, 1-pyrrolidinecarbodithioic acid, ammonium salt, NFKB inhibitor; BAY, BAY1 1-7082, NFKB inhibitor.

NFкB inhibitor, potently suppressed HIF- $1 \alpha$ and VEGF expression induced by $H$ pylori. BAY11-7082 (5 $\mu \mathrm{M})$ reversibly increased expression of the genes. These data suggest that $H$ pylori induced VEGF induction was mediated via the MAPK pathway, and partially by the NFкB pathway.

\section{PPI disturbs $H$ pylori induced signalling for angiogenesis via inactivation of MAPK ERK $1 / 2$}

Based on the previous findings that $H$ pylori infection stimulated the synthesis of angiogenic factors via MAPK ERK activation (fig 6) and that the anticancer action of PPI is fundamentally attributable to inhibition of phosphorylation of MAPK ERKl $/ 2,{ }^{27}$ we evaluated whether the antiangiogenic activity of PPI is caused by block of ERK activation. Western blot analysis with phospho-ERK antibodies was performed to determine the influence of PPI on MAPK ERK1/2 activation related to $H$ pylori induced angiogenesis (fig 7A, B). The ERK inhibitor, PD098059, and the p38 inhibitor, SB203580, decreased phosphorylation of ERKl/2. Interestingly, PPI completely attenuated phosphorylation of ERKl/2, showing stronger inhibitory activity than the ERKl/2 inhibitor PD98059 (fig 7A). These inhibitory actions of PPI against ERK phosphorylation were dose dependent (fig 7B) and maximal inhibitory activity was seen after four hours (data not shown). However, PPI did not have any effect on nuclear translocation of $\mathrm{NFKB}$, suggesting that the antiangiogenic effect of PPI seems to be independent of suppression of NFKB (fig 7C). In summary, angiogenesis induced by $H$ pylori was attenuated by PPI treatment, which was caused by inactivation of the MAPK pathway, one of principal signals for $H$ pylori induced angiogenesis.

\section{DISCUSSION}

After $H$ pylori infection, the signal transduction enzymes, MAPK ERKI/2 and NFKB, are activated and these molecules are responsible for transcriptional activation of angiogenic growth factors, including IL- 8 , HIF- $1 \alpha$, and VEGF. Increase in $H$ pylori induced angiogenic factors stimulates the recruitment and activation of endothelial cells in the gastric mucosa, resulting in significant neovascularisation of the gastric mucosal layer which can provide a vulnerable and fertile environment for carcinogenesis. Chronic gastric 

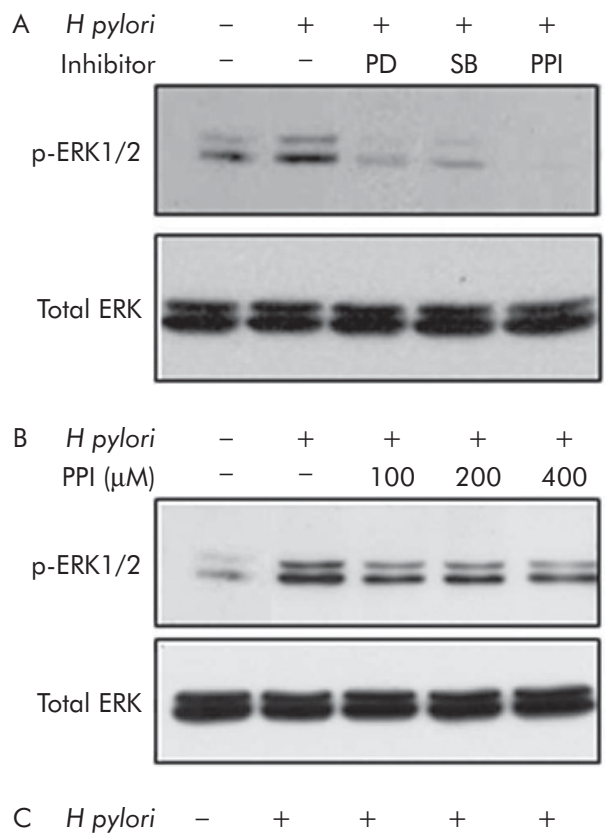

PPI $(\mu M) \quad-\quad-\quad 100 \quad 200 \quad 400$

Nucleic NFKB p65
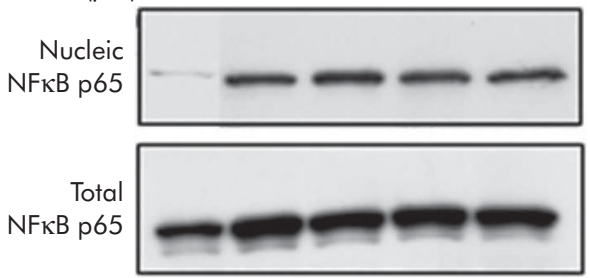

Figure 7 Deactivation of mitogen activated protein kinase (MAPK) extracellular signal regulated kinase (ERK) $1 / 2$ signalling with proton pump inhibitor (PPI). (A) To compare the inhibitory effect of MAPK inhibitors and PPI on phosphorylation of MAPK ERK1/2, AGS cells were treated with $50 \mu \mathrm{M}$ of the ERK inhibitor (PD098059), $10 \mu \mathrm{M}$ of the p38 inhibitor (SB203580), or $200 \mu \mathrm{M}$ PPI for eight hours, and then infected with Helicobacter pylori for 15 minutes. Proteins isolated from cells were subjected to immunoblotting with phospho-ERK antibodies. (B) Effect on phosphorylation of ERK at different concentrations of PPI. (C) Effect of PPI on $H$ pylori induced nuclear factor $\kappa B(N F \kappa B)$ translocation was evaluated at different concentrations of PPI. AGS cells were treated with the indicated concentrations of PPI for eight hours and inoculated with $H$ pylori for two hours. Nucleic proteins were isolated from cells and subjected to western blotting using specific NFKB antibodies. Translocation of $N F_{\kappa B} B$ was deduced from $N F_{\kappa} B$ in nuclear fractions compared with total NFKB. PD, PD098059 (ERK1/2 inhibitor), SB, SB203580 (p38 inhibitor).

inflammation triggered by $H$ pylori infection may predispose to the development and progression of gastric cancer. H pylori induced angiogenesis might contribute to this $H$ pylori associated gastric carcinogenesis along with other carcinogenic events. In this study, for the first time, we have documented the mechanistic link between $H$ pylori infection and angiogenesis, and the inhibitory effect of PPIs on $H$ pylori induced angiogenesis. PPI treatment efficiently inhibited IL-8, VEGF, and HIF- $1 \alpha$ expression in $H$ pylori infected gastric epithelial cells, which was due to inactivation of MAPK signalling induced by $H$ pylori infection (fig 7). Thus these findings have helped shed light on antiangiogenic treatment with PPIs, drugs popularly prescribed for gastro-oesophageal acid related diseases and $H$ pylori eradication. PPI therapy could be a promising protective therapeutic approach for $H$ pylori associated carcinogenesis.

Neovascularisation, the development of new blood vessels from existing endothelial precursors, is a general physiological mechanism critically involved in the normal repair process and in the pathogenesis of inflammatory and ulcerative epithelial lesions as well as malignant tumour growth, and even tumour metastasis. ${ }^{30}{ }^{31}$ Among the proangiogenic factors known, VEGF represents one of the most potent stimuli of neoangiogenesis. Although previous studies found that enhanced VEGF gene expression contributed to the healing of peptic lesions in the stomach, ${ }^{32}{ }^{33}$ here we demonstrated that $H$ pylori infection stimulated upregulation of proangiogenic factors and increased infiltration of blood vessels in the mucosa layer, which might be positively associated with propagation of gastric inflammation as well as gastric carcinogenesis after $H$ pylori infection. Several investigations suggested that $H$ pylori infection stimulates host VEGF-A gene expression and $H$ pylori induced angiogenesis may play a critical role in the development of gastric cancer. ${ }^{34-36}$ Gastric adenocarcinomas frequently showed high levels of VEGF expression, ${ }^{34}{ }^{35}$ and neutralisation of circulating VEGF with specific VEGF antibodies potently reduced the growth of gastric cancer. ${ }^{36}$ Potent stimulators of angiogenesis related to $H$ pylori infection, $H$ pylori VacA toxin, ${ }^{37}$ reactive oxygen species synthesised from neutrophils or macrophages, ${ }^{38}$ CagA pathogenicity islands, ${ }^{12}{ }^{14}$ and lipopolysaccharide have been reported, and several pathways linking the bacterium to host angiogenesis have been revealed. A wider spectrum of genes induced by $H$ pylori in gastric epithelium was identified by high throughput analysis of cDNA microarray analysis, and most of these genes are responsible for angiogenesis and tumour invasion stimuli, such as ADAM series, IL-8, VEGF, integrins, VCAM-1, ICAM-1, E-selectin, GRO- $\alpha$, and IL- $6 .{ }^{12}{ }^{14}$

The PPI PPZ, a substituted 2-pyridyl methyl/sulfinyl benzimidazole derivative, is a prodrug requiring protonation under acidic conditions for functional activation, accumulates selectively in the acidic gastric luminal space, and ultimately inhibits acid secretion by covalent binding with cysteine residues on the $\alpha$ subunit of $\mathrm{H}^{+} / \mathrm{K}^{+}$-ATPase. These PPIs have been universally used with antibiotics for the eradication of $H$ pylori and several types of acid related diseases, including gastro-oesophageal reflux diseases, peptic ulcer disease, and Zollinger-Ellison syndrome. ${ }^{19}{ }^{20}$ Increased gastric $\mathrm{pH}$ by PPIs stimulates resting $H$ pylori to activate metabolically and thus $H$ pylori are more susceptible to antibiotics. ${ }^{21-26}$ Apart from enhancing the susceptibility of $H$ pylori to antibiotics, we found, for the first time, that PPIs can directly influence host angiogenesis induced by $H$ pylori. Previously, we reported that PPIs had a strong inhibitory effect on phosphorylation of MAPK ERKl/2 and its administration showed anticancer activity in the xenograft nude mice model. ${ }^{27}$ These inhibitory actions of the drug against ERK phosphorylation also involved suppression of $H$ pylori induced host angiogenesis. Blocking of $\mathrm{H}^{+}$by PPIs caused an increase in extracellular $\mathrm{pH}$ (gastric lumen) and a decrease in intracellular $\mathrm{pH}$. The increased $\mathrm{pH}$ of the extracellular space may improve the hypoxic microenvironment surrounding gastric epithelial cells and interrupt $H$ pylori induced intracellular signalling via inhibition of MAPK activation. Compared with inhibitors of ERKl/2 or p38, PPZ showed stronger inhibitory activities than these (fig 7), in a dose dependent manner. However, the influence of this drug on NFKB transcriptional activation was minor (fig 7).

In conclusion, we have shown that considerable angiogenic activities were stimulated in the gastric mucosa after $H$ pylori infection, for which increasing proangiogenic growth factors from gastric epithelial cells were responsible. PPIs could have significant inhibitory activities against $H$ pylori associated angiogenesis. Therefore, the current data indicate that as $H$ pylori infection causally promoted host angiogenesis, which has been attributed to either augmented inflammation or enhanced carcinogenesis, PPIs could be potentially used for inhibition of $H$ pylori provoked angiogenesis. 


\section{ACKNOWLEDGEMENTS}

This work was supported by grants of the Korea Heath 21 R\&D Project, Ministry of Health and Welfare (01-PJ10-PG6-01GN140007).

\section{Authors' affiliations}

M Yeo, D-K Kim, S U Han, Y B Kim, Y K Cho, J H Kim, S W Cho, K-B

Hahm, Genomic Research Centre for Gastroenterology and Department of Gastroenterology, Ajou University School of Medicine, Suwon, Korea J E Lee, DNA Link, Seoul, Korea

Conflict of interest: None declared.

\section{REFERENCES}

1 Peek RM Jr, Blaser MJ. Helicobacter pylori and gastrointestinal tract adenocarcinomas. Nat Rev Cancer 2002;2:28-37.

2 Stolte M, Bayerdorffer E, Morgner A, et al. Helicobacter and gastric MALT lymphoma. Gut 2002;50:19-24.

3 Scheiman JM, Cutler AF. Helicobacter pylori and gastric cancer. Am J Med 1999; 106:222-6.

4 Touati E, Michel V, Thiberge JM, et al. Chronic Helicobacter pylori infections induce gastric mutations in mice. Gastroenterology 2003;124:1408-19.

5 Bagchi D, Bhattacharya G, Stohs SJ. Production of reactive oxygen species by gastric cells in association with Helicobacter pylori. Free Radic Res 1996;24:439-50

6 Maeda S, Yoshida H, Mitsuno Y, et al. Analysis of apoptotic and antiapoptotic signalling pathways induced by Helicobacter pylori. Gut 2002;50:771-8.

7 Gupta RA, Polk DB, Krishna U, et al. Activation of peroxisome proliferatoractivated receptor gamma suppresses nuclear factor kappa B-mediated apoptosis induced by Helicobacter pylori in gastric epithelial cells. J Biol Chem 2001;276:31059-66.

8 Betten A, Bylund J, Cristophe T, et al. A proinflammatory peptide from Helicobacter pylori activates monocytes to induce lymphocyte dysfunction and apoptosis. J Clin Invest, 2001;108:1221-8.

9 Allen LA. Modulating phagocyte activation: the pros and cons of Helicobacter pylori virulence factors. J Exp Med 2000;191:1451-4.

10 Crawford HC, Krishna US, Israel DA, et al. Helicobacter pylori strain-selective induction of matrix metalloproteinase-7 in vitro and within gastric mucosa. Gastroenterology 2003;125:1125-36.

11 Kitadai $Y$, Sasaki A, lto $M$, et al. Helicobacter pylori infection influences expression of genes related to angiogenesis and invasion in human gastric carcinoma cells. Biochem Biophys Res Commun 2003;311:809-14.

12 Cox JM, Clayton CL, Tomita T, et al. cDNA array analysis of cag pathogenicity island-associated Helicobacter pylori epithelial cell response genes. Infect Immun 2001;69:6970-80

13 Strowski MZ, Cramer T, Schafer G, et al. Helicobacter pylori stimulates host vascular endothelial growth factor-A (vegf-A) gene expression via MEK/ERK dependent activation of Spl and Sp3. FASEB J 2004;18:218-20.

14 Innocenti M, Thoreson AC, Ferrero RL, et al. Helicobacter pylori-induced activation of human endothelial cells. Infect Immun 2002;70:4581-90.

15 Franceschi F, Genta RM, Gasbarrini A, et al. Helicobacter pylori infection and expression of the angiogenic factor platelet-derived endothelial cell growth factor by pre-neoplastic gastric mucosal lesions and gastric carcinoma. Dig Liver Dis 2002;34:621-5.

16 Sachs G, Shin JM, Briving C, et al. The pharmacology of the gastric acid pump: the $\mathrm{H}^{+}, \mathrm{K}^{+}$-ATPase. Ann Rev Pharmacol Toxicol 1995;35:277-305.
17 Scott DR, Helander HF, Hersey SJ, et al. The site of acid secretion in the mammalian parietal cell. Biochim Biophys Acta Bio-Membr 1993; 1146:73-80.

18 Horn J. The proton-pump inhibitors: similarities and differences. Clin Ther 2000;22:266-80.

19 Sachs G. Proton pump inhibitors and acid-related diseases. Pharmacotherapy 1997; 17:22-37.

20 Fitton A, Wiseman L. Pantoprazole. A review of its pharmacological properties and therapeutic use in acid-related disorders. Drugs 1996:51:460-82.

21 Iwahi T, Satoh H, Nakao M, et al. Lansoprazole, a novel benzimidazole proton pump inhibitor, and its related compounds have selective activity against Helicobacter pylori. Antimicrob Agents Chemother 1991;35:490-6.

22 Nakao M, Malfertheiner P. Growth inhibitory and bactericidal activities of lansoprazole compared with those of omeprazole and pantoprazole against Helicobacter pylori. Helicobacter 1998;3:21-7

23 Hirai M, Azuma T, Ito S, et al. A proton pump inhibitor, E3810, has antibacterial activity through binding to Helicobacter pylori. J Gastroenterol 1995;30:461-4.

24 Tsuchiya M, Imamura L, Park JB, et al. Helicobacter pylori urease inhibition by rabeprazole, a proton pump inhibitor. Biol Pharm Bull 1995;18:1053-6.

25 McGowan CC, Cover TL, Blaser MJ. The proton pump inhibitor omeprazole inhibits acid survival of Helicobacter pylori by a urease-independent mechanism. Gastroenterology 1994:107:1573-8.

26 Mauch F, Bode G, Malfertheiner P. Identification and characterization of an ATPase system of Helicobacter pylori and the effect of proton pump inhibitors. Am J Gastroenterol 1993;88:1801-2.

27 Yeo M, Kim DK, Kim YB, et al. Selective induction of apoptosis with proton pump inhibitor in gastric cancer cells. Clin Cancer Res 2004;10:8687-96.

28 Dixon MF, Genta RM, Yardley JH, et al. Classification and grading of gastritis, the updated Sydney system. Am J Surg Pathol 1996;20:1161-81.

29 Jaffe EA, Nachman RL, Becker CG. Culture of human endothelial cells derived from umbilical veins. Identification by morphologic and immunologic criteria, J Clin Invest 1973;52, 2745-56.

30 Jordan MA, Wilson L. Microtubules as a target for anticancer drugs. Nat Rev Cancer 2004;4:253-65.

31 Blagosklonny MV. Antiangiogenic therapy and tumor progression. Cancer Cell 2004;5:13-17.

32 Baatar D, Jones MK, Tsugawa K, et al. Esophageal ulceration triggers expression of hypoxia-inducible factor-1 alpha and activates vascular endothelial growth factor gene: implications for angiogenesis and ulcer healing. Am J Pathol 2002;161:1449-57.

33 Jones MK, Kawanaka H, Baatar D, et al. Gene therapy for gastric ulcers with single local injection of naked DNA encoding VEGF and angiopoietin-1. Gastroenterology 2001;121:1040-7

34 Takahashi Y, Cleary KR, Mai M, et al. Significance of vessel count and vascular endothelial growth factor and its receptor (KDR) in intestinal-type gastric cancer. Clin Cancer Res 1996;2:1679-84.

35 Maeda K, Kang SM, Onoda N, et al. Vascular endothelial growth factor expression in preoperative biopsy specimens correlates with disease recurrence in patients with early gastric carcinoma. Cancer 1999:86:566-71.

36 Kanai $\mathrm{T}$, Konno $\mathrm{H}$, Tanaka $\mathrm{T}$, et al. Anti-tumor and anti-metastatic effects of human-vascular-endothelial-growth-factor-neutralizing antibody on human colon and gastric carcinoma xenotransplanted orthotopically into nude mice. Int J Cancer 1998;77:933-6.

37 Caputo R, Tuccillo C, Manzo BA, et al. Helicobacter pylori VacA toxin upregulates vascular endothelial growth factor expression in MKN 28 gastric cells through an epidermal growth factor receptor-, cyclooxygenase-2dependent mechanism. Clin Cancer Res 2003;9:2015-21.

38 Park JH, Kim TY, Jong HS, et al. Gastric epithelial reactive oxygen species prevent normoxic degradation of hypoxia-inducible factor-lalpha in gastric cancer cells. Clin Cancer Res 2003:9:433-40. 